\title{
Cleavable and thermo-responsive hybrid
}

\section{nanoparticles for on-demand drug delivery}

Isabel Ortiz de Solorzano ${ }^{\dagger \xi^{*}}$, Teresa Alejo ${ }^{\dagger *}$, Miriam Abad", Carlos Bueno-Alejo ${ }^{\dagger}$ Gracia Mendoza ${ }^{\dagger \ddagger}$, Vanesa Andreu ${ }^{\dagger \ddagger}$, Silvia Irusta ${ }^{\dagger \$ s}$, Victor Sebastian ${ }^{\dagger \$}$, Manuel Arruebo ${ }^{\dagger \dagger \xi}$

'Department of Chemical Engineering. Aragon Institute of Nanoscience (INA), University of Zaragoza, Campus Río Ebro-Edificio I+D, C/ Poeta Mariano Esquillor S/N, 50018-Zaragoza, Spain

†Aragon Health Research Institute (IIS Aragón), 50009 Zaragoza, Spain

${ }^{\S}$ Networking Research Center on Bioengineering, Biomaterials and Nanomedicine, CIBER-BBN, 28029-Madrid, Spain

"Departamento de Química Orgánica, Facultad de Ciencias, Instituto de Ciencia de Materiales de Aragón (ICMA), Universidad de Zaragoza-CSIC, 50009 Zaragoza, Spain

\section{Corresponding Author}

Isabel Ortiz de Solórzano

C/ Poeta Mariano Esquillor S/N,

Campus Río Ebro-Edificio I+D, 50018-Zaragoza, Spain

*Email: isaortiz@unizar.es Tel.: +34 876555428.

Keywords: Thermosensitive polymer, cleavable nanoparticles, bupivacaine, drug delivery, photothermal; NIR. 
https://daneshyari.com/article/8947533 